\title{
Increase of resting muscle stiffness, a less considered component of age-related skeletal muscle impairment
}

\author{
Lorenzo Marcucci $(1,2,3)$, Carlo Reggiani $(1,2,4)$
}

(1) Department of Biomedical Sciences, Padova University, Padova, Italy; (2) Center for Mechanics of Biological Materials, Padova University, Padova, Italy; (3) Center for Biosystems Dynamics Research, RIKEN, Suita, Osaka, 565-0874, Japan; (4) Science and Research Centre Koper, Institute for Kinesiology Research, Koper, Slovenia

This article is distributed under the terms of the Creative Commons Attribution Noncommercial License (CC BY-NC 4.0) which permits any noncommercial use, distribution, and reproduction in any medium, provided the original author(s) and source are credited.

\begin{abstract}
Elderly people perform more slowly movements of everyday life as rising from a chair, walking, and climbing stairs. This is in the first place due to the loss of muscle contractile force which is even more pronounced than the loss of muscle mass. In addition, a secondary, but not negligible, component is the rigidity or increased stiffness which requires greater effort to produce the same movement and limits the range of motion of the joints. In this short review, we discuss the possible determinants of the limitations of joint mobility in healthy elderly, starting with the agedependent alterations of the articular structure and focusing on the increased stiffness of the skeletal muscles. Thereafter, the possible mechanisms of the increased stiffness of the muscletendon complex are considered, among them changes in the muscle fibers, alterations of the connective tissue components, i.e., extracellular matrix (ECM), aponeurosis, tendon and fascia, and remodeling of the neural pattern of muscle activation that increases antagonist co-activation.
\end{abstract}

Key Words: Joint mobility, muscle stiffness, aging, connective tissue, modeling

Eur J Transl Myol 30 (2): 223-233, 2020

The decrease of mass and strength are the hallmarks of the skeletal muscle aging. The decrease in muscle contractile performance has an impact on mobility of the elderly people, sets limitations to independent life and increases the risk of falls, but further limitations to the mobility of elderly people come also from other factors as the reduction of range of motion of the joints (see Figure 1). Joint mobility declines as age increases, with a more rapid reduction during the ninth decade. The range of motion is reduced and more force is required for movement. ${ }^{1}$ Not all joints and not all movements are equally impaired. For example, only a limited decline is reported for knee flexion-extension, while a greater reduction (up to 20\%) is reported for hip extension, ${ }^{2}$ and for calf dorsiflexion. ${ }^{3,4}$ In the upper part of the body pronounced reduction of mobility of shoulders occurs in elderly, ${ }^{5,6}$ and reduced flexibility of the neck is very common, particularly in elderly males (see for review and meta-analysis Pan et al. ${ }^{7}$ ). Several factors contribute to the decrease in joint mobility, among them changes in mechanical properties of the joints, ${ }^{8}$ of the fascia, ${ }^{9}$ and of the muscle-tendon complex. ${ }^{10,11}$ Alterations of the nervous commands can also contribute to reduce joint flexibility, e.g., with increased antagonist co-activation. ${ }^{12}$

\section{Age-dependent changes in mechanical properties of the joints}

The age-related limitation of joint mobility (LJM) recognizes several determinants (see for a review Abate et al. ${ }^{8}$ ), among them:

i) Thickening and structural alterations of the joint capsule. Parts of the capsule become fibrocartilaginous and pressure resistant, with accumulation of glycosaminoglycans and, in some cases, type II collagen, typical of cartilage (see e.g. Ralphs and Benjamin). ${ }^{13}$ Age related alterations involve also the fibro-cartilages associated with the capsule, as the knee menisci (see for a review, Tsujii et al. ${ }^{14}$ );

ii) Stiffening of the ligaments, which can nowadays be evaluated with shear wave elastography. A recent study has shown the stiffening of coraco-humeral ligament in elderly and demonstrated a strong correlation with the reduction of shoulder range of motion (ROM) in abduction and extra-rotation. ${ }^{15}$ Reduction of blood flow to the capsule and the ligaments seems to be an important pathogenic factor in determining the age-related alterations. ${ }^{16,17}$ At molecular level, the main biochemical abnormality, 


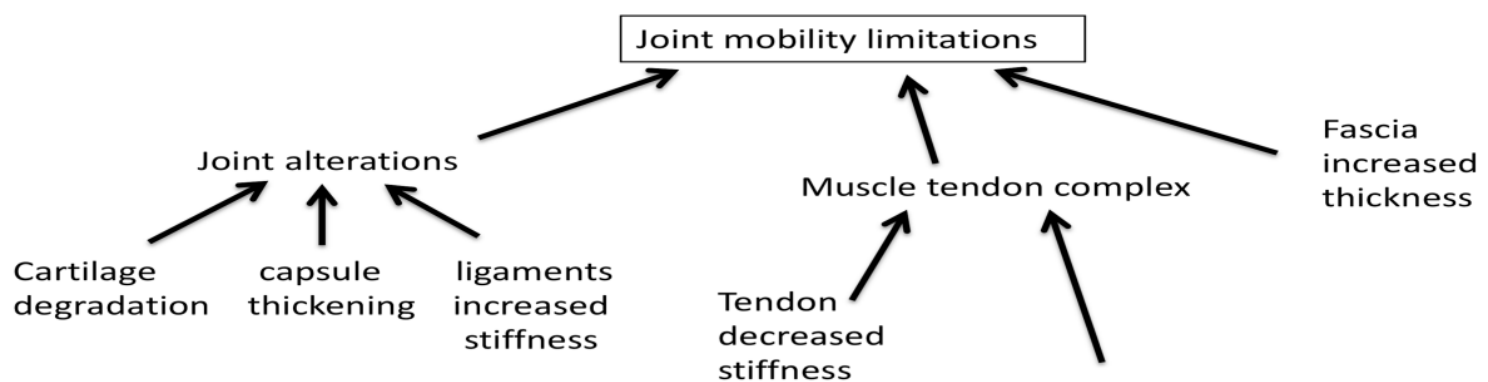
stiffness

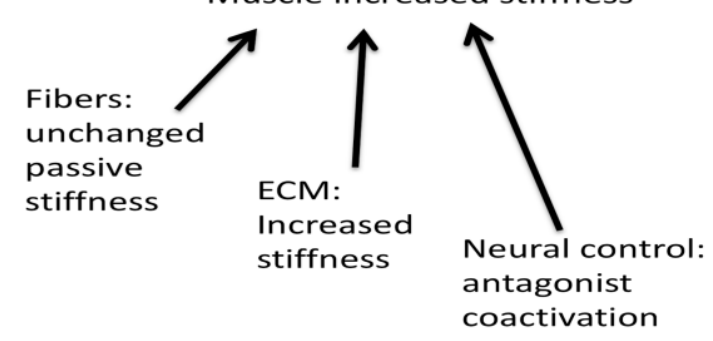

Fig 1. Schematic outline of the factors playing a role in determining the limitation of the joint mobility in the elderly. Changes in resting muscle mechanical properties play an essential role.

common to aging and diabetes, is the non-enzymatic glycosylation of collagen, with advanced glycation end product (AGE) formation, which in turn leads to an increase of collagen cross-links;

iii) Beside the involvement of articular connective structures, the age-related changes affect both the structural and matrix composition of articular cartilage (see for a review Toh et al. ${ }^{18}$ ) and the properties and functions of chondrocytes which loose proliferation rate and matrix biosynthetic activity (aggrecan and type-II collagen). Proteoglycan production, glycosaminoglycan (GAG) content, matrix metalloproteinase (MMP)-2 activity are significantly reduced while AGE accumulation is increased in articular cartilage of healthy elderly.

In this regard, it is important to note that osteoarthritis is not an inevitable consequence of aging and cannot be considered only a simple "wearing out" of the joints. The aging-related changes in the joints can be distinguished from those due to disease, see for a review Loeser. ${ }^{19}$

\section{Age-related alterations of muscle-tendon complex}

The stiffness of the muscle-tendon complex determined in situ increases with aging and this is mainly attributed to an increased stiffness of the muscles, as tendons undergo to an increase of compliance (see also below). ${ }^{20,21}$ Studies on ex vivo isolated muscles of rodents have shown that the length-passive tension curves are more steep in old than in young animals, thus confirming an age-dependent increase of stiffness. ${ }^{22,23}$ Accordingly, determination of passive stiffness with sinusoidal oscillations confirmed that resting soleus is stiffer in 23 mo old rats than in young 3 mo rats (Figure 2). ${ }^{24}$ There are, however, contrasting reports of unchanged passive stiffness in hind limb muscles of old $(33 \mathrm{mo})$ rat compared to young adult $(7 \mathrm{mo}) .{ }^{25}$ The stiffness of human muscle in situ has been determined with different approaches. Stiffness at rest has been evaluated by measuring the resistance to stretch and found to be increased in elderly in several studies (see e.g. Blanpied and Smidt ${ }^{26}$ comparing plantar flexors of young and old women. Palmer and Thompson ${ }^{27}$ comparing hamstrings in young and old men, or with quick release movements (see e.g. Ochala et al. ${ }^{28}$ comparing plantar flexors in old and young men, Valour an Pousson ${ }^{29}$ comparing elbow flexors at different ages). Sobolewski and co-workers have measured not only stiffness during a $10^{\circ}$ passive dorsal flexion of the ankle, ${ }^{30}$ but also stress relaxation and found that the stiffness was higher while stress relaxation was lower in elderly men (67 y) compared to young (24 y). In contrast, recent determinations based on shear wave elastography have found a decreased stiffness of leg muscles in shortened position and a preserved, but not increased, stiffness in stretched position in older (77-94 y) compared to young (20-35y) subjects. ${ }^{31}$ Importantly, the age-related increase in stiffness of the muscle-tendon complex has been considered relevant to the preservation of eccentric force in the elderly. The earlier observations that, in the elderly, the force generated in eccentric contraction of leg extensor muscles is reduced less than force developed during isometric and concentric contractions date back to ' $90 \mathrm{~s} .{ }^{32,33}$ As summarized in the review by Roig and coworkers, ${ }^{34}$ the preservation of eccentric force and/or torque was confirmed by several subsequent studies in different age groups, different joints and different joint angular rotation velocities. The 

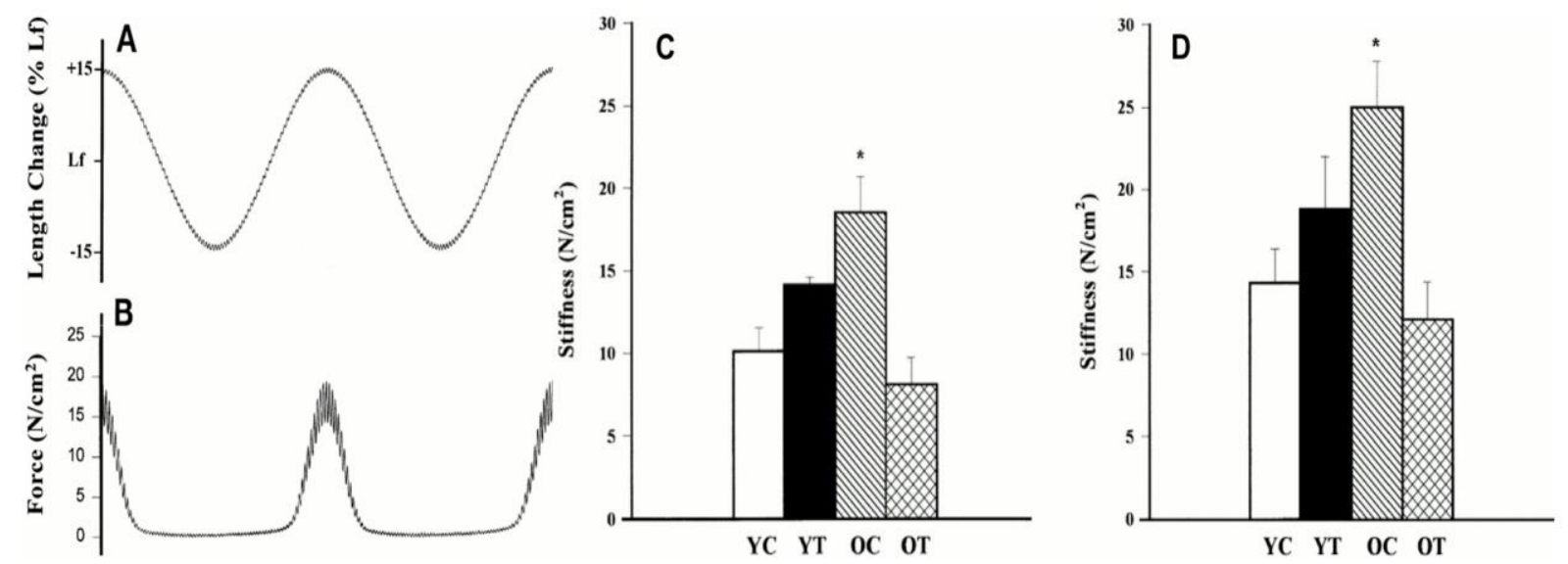

Fig 2. Determination of mechanical stiffness of isolated Soleus muscle of the rat at rest. A: length changes of soleus muscle lengthened and shortened $\pm 15 \%$ of optimal fiber length $\left(L_{f}\right)$ at $1 \mathrm{~Hz}$ with smaller $\left( \pm 0.5 \% L_{f}\right)$ oscillation at $75 \mathrm{~Hz}$ superimposed. B: corresponding force changes of soleus muscle during changes in muscle length. $C$ : passive stiffness $\left(\mathrm{N} / \mathrm{cm}^{2}\right)$ of rat soleus at $111 \%$ optimal length from young (3 mo) control (YC), young (3 mo) trained $(Y T)$, old $(23 \mathrm{mo})$ control $(O C)$, and old $(23$ mo) trained $(O T)$ groups. D: the same at 115\% optimal length. Means \pm SE. (Reproduced with permission from Gosselin et al 1998, ${ }^{24}$ modified. License numbers 4800981198132 and 4803160486433$)$

search for a mechanistic explanation has considered multiple alternatives from increased antagonist coactivation, ${ }^{35,36}$ to increased whole muscle stiffness with a possible relevant contribution of ECM and connective tissue to an intrinsic alterations of the contractile mechanism at cross bridge level. ${ }^{37-39}$ It is worth to recall that the preservation of eccentric strength in the elderly has been considered of interest for training and rehabilitation. Eccentric contractions have a better efficiency than concentric contraction (i.e. muscle force is achieved at a lower metabolic cost) and thus eccentric contractions might offer a possibility to increase the intensity of resistance training (greater loads) in elderly with limited muscle capacity. ${ }^{40,41}$ Beside the possible specific contribution to the preservation of eccentric force, the increase of muscle stiffness with aging is relevant for the motor performance of elderly people and enjoys a strong experimental support. It is, therefore, worth to discuss analytically the possible determinants. The whole muscle stiffness depends on the mechanical properties of muscle fibers and ECM, which includes endomysium in direct contact with individual fibers, perimysium, which surrounds fiber bundles or fascicles and epimysium which covers the whole muscle belly. In addition, the mechanical properties of the tendon and of the aponeurosis give a significant contribution to the elasticity of the muscle-tendon complex. Finally, if the muscle-tendon complex is investigated in situ, the motor neuron discharge needs to be taken into account even at rest.

Changes in resting mechanical properties of muscle single fibers with age

While a large body of experimental evidence is available regarding the age-dependent alterations of the contractile properties of single muscle fibers (see for example Trappe et al. ${ }^{42}$ or D'Antona et al. ${ }^{43}$ ), much less data are available on their mechanical properties at rest. The few data available are conflicting as a recent paper by Lim et al. ${ }^{44}$ has shown an increase of passive stiffness in single fibers from elderly humans (average $79 \mathrm{y}$ ) compared to those of young subjects ( $25 \mathrm{y}$ ), while Wood et al. ${ }^{23}$ have not found any difference in single muscle fibers from adult (8-10 mo) and old (28-30 mo) mice. These contrasting findings propose alternative explanations for the age-dependent increase of muscle stiffness. The results of Lim and coworkers suggest a major contribution given by the change in mechanical properties of muscle fibers, ${ }^{44}$ while the results of Wood et al. ${ }^{23}$ identify ECM and connective tissues as the only responsible. A comparison is possible with the increase of stiffness of skeletal muscles of patients with spasticity due to lesions of the upper motor neuron. The patients with spasticity display an increased muscle rigidity, which is partly caused by an exaggerated stretch reflex and partly by altered mechanical properties of the muscle at rest. In particular, the isolated muscle fibers of the patients are characterized by shorter slack length and increased resting tension..$^{45,46}$ It is widely accepted that mechanical properties of individual muscle fibers at rest are determined by titin, ${ }^{47}$ with minor contribution of other extra-myofibrillar proteins as desmin (see also the review of Schiaffino and Reggiani). ${ }^{48}$ Thus, a change in passive tension might be attributed to the presence of specific titin isoforms. Experimental evidences show a link between long titin isoforms and low passive tension in slow fibers and shorter isoforms and higher passive tension in fast fibers of rabbit, ${ }^{47,49,50}$ and rat. ${ }^{51}$ In contrast, 

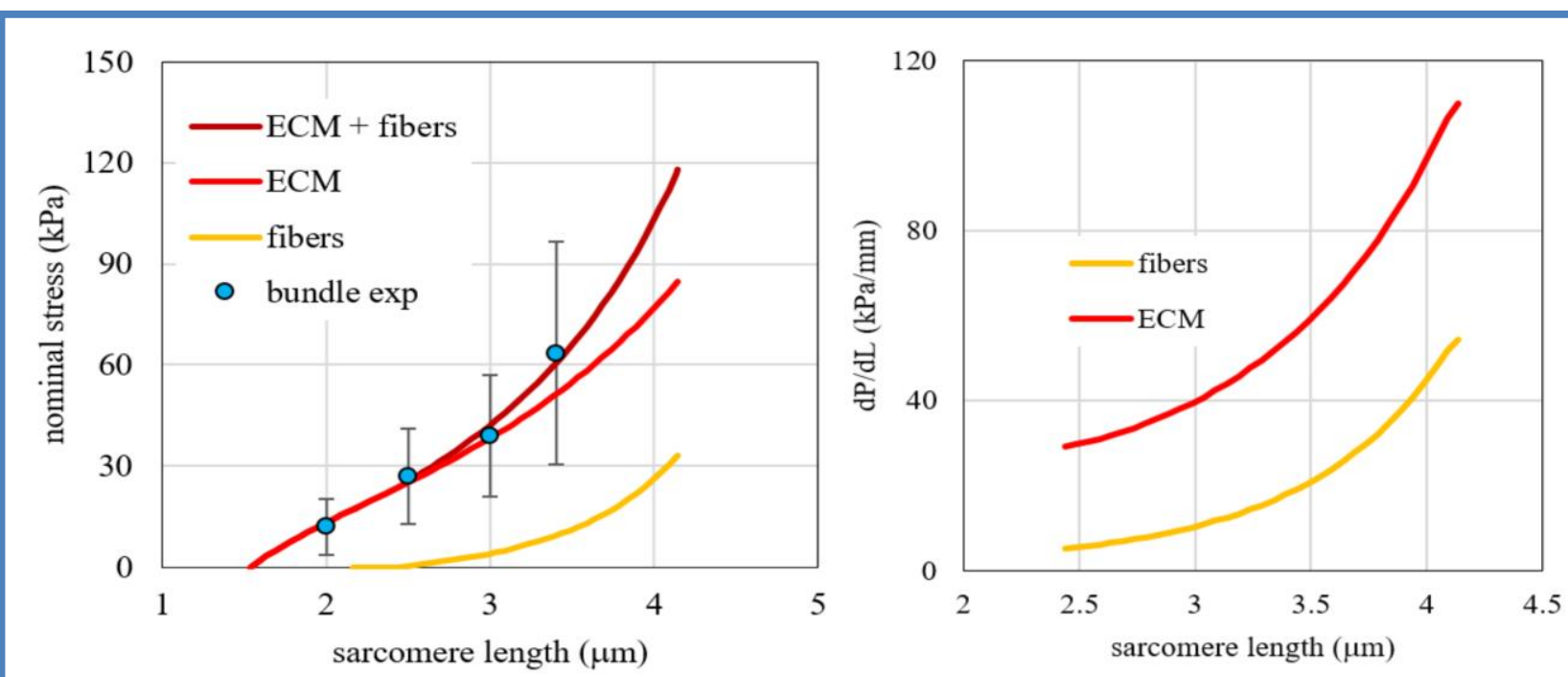

Fig 3. Contributions of fibers and Extracellular Matrix elasticity to the elasticity of a muscle fiber bundle. Left panel: Resting tension vs sarcomere length of fiber bundles (blue dot experimental data, means and SD, brown curve mathematical interpolation) is shown with the component due to muscle fibers (yellow curve) and the component due to ECM (red curve). Right panel: tangent stiffness vs. sarcomere length for fibers (yellow curve) and for ECM (red curve). The latter is obtained from the analytic calculation of the specific contribution of ECM to passive tension (see left panel) (from Marcucci et al 2019). ${ }^{60}$

no difference in resting mechanical properties has been found comparing slow and fast single fibers of healthy human muscles. ${ }^{46,50}$ An explanation for the possible difference observed in relation with aging, ${ }^{44}$ or spasticity, ${ }^{45,46}$ can be found in post-translational modifications of titin (phosphorylation and oxidative modifications) $)^{52}$ or in calcium dependent changes of the titin properties. ${ }^{53}$ Alternatively, the possibility that remnants of extracellular collagen molecules are still present in dissected single fibers and thus contribute to their mechanical properties has been considered. ${ }^{44,46}$

\section{Changes of muscle extracellular matrix with age}

Muscle fibers are embedded in a connective tissue network which consists of three layers: the endomysium surrounding muscle fibers, the perimysium surrounding fascicles, and the epimysium surrounding the entire muscle (for a comprehensive review, see Kjaer). ${ }^{54}$ The collagen fiber network of the ECM is a major contributor to the passive force, ${ }^{55}$ in addition to its role in force transmission during contraction. ${ }^{56,57}$ Due to its structural arrangement in close relation with muscle fibers, the determination of the mechanical properties of ECM is extremely difficult. An approach based on comparison of mechanical properties of bundles (fibers and ECM) and single fibers has been developed by Meyer and Lieber, ${ }^{58,59}$ and applied to age-dependent alterations of murine muscle by Wood et al. ${ }^{23}$ and to human skeletal muscles by Marcucci et al. ${ }^{60}$ Both the results of Wood et al. $^{23}$ and Marcucci et al. ${ }^{60}$ have shown that ECM is responsible for a substantial proportion of resting stiffness and passive tension. In aged mice muscle, at sarcomere length around $3 \mu \mathrm{m}$, passive tension and stiffness are double in fiber bundles compared to single fibers dissected from old but not from young mice. ${ }^{23}$ The biomechanical difference was tentatively related to a change in ECM composition (accumulation of AGE, increase of hydroxiproline content) as the histologically determined amount of ECM was unchanged. ${ }^{23}$ The accumulation of AGE and the increase of collagen content has been reported also by Kragstrup et al. ${ }^{61}$

A similar analysis of resting stiffness and tension in muscle fiber bundles from elderly humans, ${ }^{60}$ yields values of tangent stiffness modulus of ECM higher than those of single fibers (Figure 3). This further demonstrates the major role played by ECM in determining the biomechanical properties of skeletal muscles of elderly at rest. Uniaxial stretching tests have been carried out on epimysium dissected from tibialis anterior of rats of different ages and have shown that, the epimysium from old rats is much stiffer than that of the young rats with no detectable differences in the ultrastructure and thickness of the epimysium or size of the collagen fibrils. ${ }^{62}$

Age-related changes in mechanical properties of tendons and aponeuroses

In contrast with the increase in stiffness of the tendonmuscle complex, there is evidence that tendons undergo to reduction of stiffness with aging. This was first shown by Onambele et al. ${ }^{21}$ who compared Achilles Tendon in three groups of age $24 \mathrm{y}, 46 \mathrm{y}$ and $60 \mathrm{y}$ (see figure 4) and recently confirmed by Delabastita et al. ${ }^{63}$ who published a systematic literature review. Similar reductions have been reported in very old people (comparing 65 y to 83 y) for the patellar tendon by Eriksen et al. ${ }^{64}$ and by Hsiao 

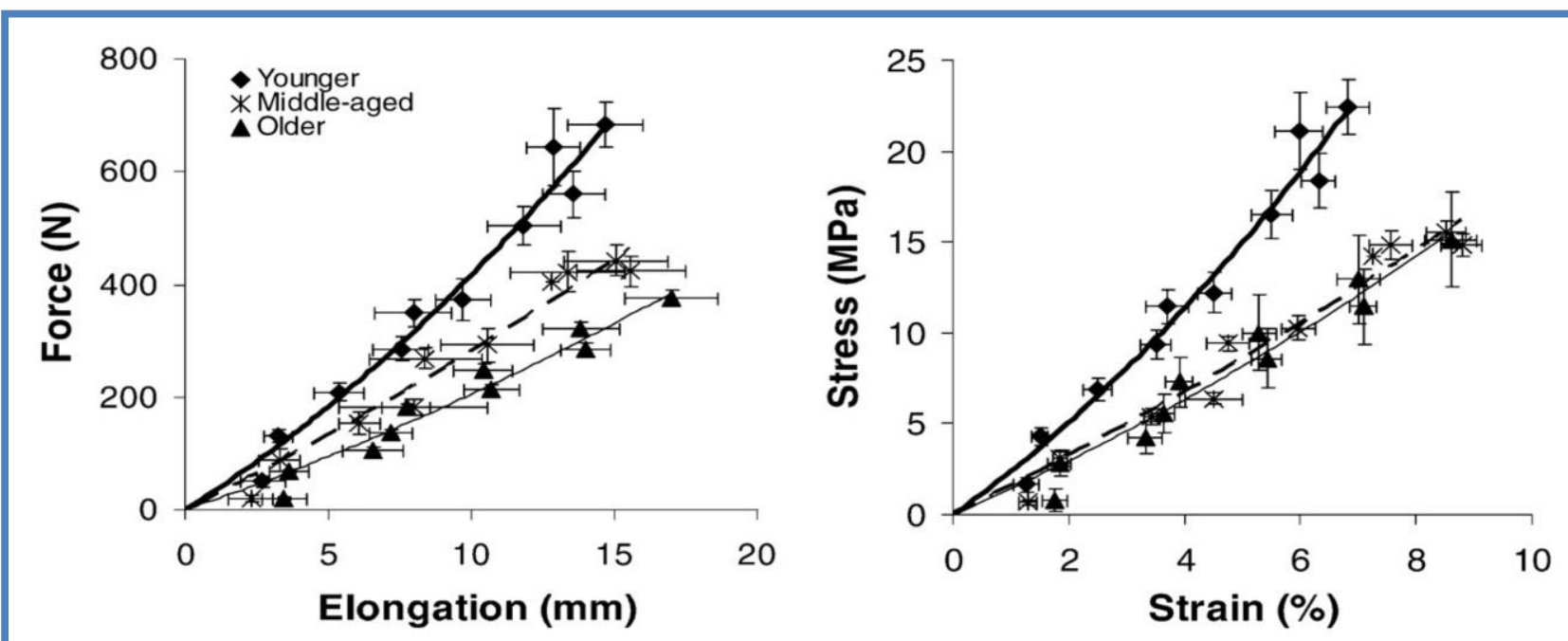

Fig. 4. Human Gastrocnemius tendon force-elongation curves (left) and stress-strain curves (right) in three distinct age groups : young (24 y), middle age $46 y$ ) and old (68y). Data are means and SE. The reduction in elasticity in the elderly is easily detectable. (Reproduced with permission from Onambele et al. 2006, ${ }^{21}$ license number 4802321103155.)

et al. ${ }^{65}$ who compared, using shear wave elastography, a group of elderly (60-70 y) with two groups of younger subjects. In the upper limb similar age-related biochemical and mechanical alterations have been reported for the tendons of biceps, supraspinatus and subscapularis involved in the structure and function of the rotator cuff. ${ }^{66}$ In vitro studies confirm that tendons become weaker or more compliant with aging (see for example Vogel ${ }^{67}$ Nakagava et al. ${ }^{68}$, Dressler et al. ${ }^{69}$ ). Aponeuroses undergo to age-dependent changes similar to those observed in the tendons. Aponeuroses are connective tissue sheets, continuous with tendons, and providing a broad insertion point for muscle fibers. The collagen organization is similar to that of the tendons, and the few stiffness measurements indicate values and trend of changes not far from those measured in the tendon. For example, measurements of calf muscle aponeuroses and Achilles tendon by shear wave elastography have shown a reduced stiffness (lower shear wave speed) in adult and old men compared to young. ${ }^{70}$ At cellular and molecular level, the main aging-related changes of the tendons are the decreased cellular density, the reduced turnover of the matrix protein, the increase of AGE and possible reduction in size and density of collagen I fibrils (see for reviews Reeves et al. ${ }^{10}$ and Svensson et al. ${ }^{71}$ ). Although the changes of tendon properties might be attributed to a reduced mechanical loading, it is still debated whether resistance training is able to reverse the change. ${ }^{64,71}$

\section{Changes of structural and mechanical properties of the fascia with aging}

Fascia is a web of connective tissue that wraps around all muscles, contributes to transmitting the muscular forces at a distance and connects the different segments of the limbs, thus affecting joint mobility. ${ }^{72,73}$. The mechanical properties of deep fasciae strongly affect muscular actions. Determinations of the mechanical properties of temporal fascia samples have shown an increased stiffness in older compared to younger subjects. ${ }^{74}$ Correlations between lumbar and hamstring flexibility with thickness of the fascia have demonstrated that increased thickness of the fascia is associated with reduced flexibility in older (69 y) compared to young (22 y) women. ${ }^{9}$

\section{Increased antagonist coactivation in the elderly}

Antagonist co-activation is a factor which should not be overlooked when considering the reduction of joint range of motion and the increased stiffness in the elderly. There is a marked difference in the strategy adopted by central nervous system to generate a given torque in an elderly compared to a young person. While in a young person, the torque at a joint is obtained by optimal scaling of the activation of the agonist and the concurrent activity of the antagonist muscles, in an elderly the same torque is obtained with virtually complete activation of the agonists counteracted by a disproportionate co-activation of the antagonist muscles. This strategy is likely adopted by the elderly in order to stiffen and stabilize the joints and compensate for loss of muscle force and for ligament laxity (see Hortobagyi and Devita for a review and a discussion of possible mechanisms). ${ }^{36}$ The antagonist coactivation is typically present during voluntary movement and automatic movement as locomotion but can possibly be present at rest and contribute to limitation of passive joint mobility. Available data show how the increase coactivation of knee flexors can impair the action of the quadriceps to produce knee extension in elderly (69 y) women. ${ }^{35}$ In contrast, there are data showing that antagonist (triceps) coactivation does not contribute to the increased stiffness of the elbow flexors (biceps brachii) in elderly (67 y) subjects. ${ }^{29}$ 


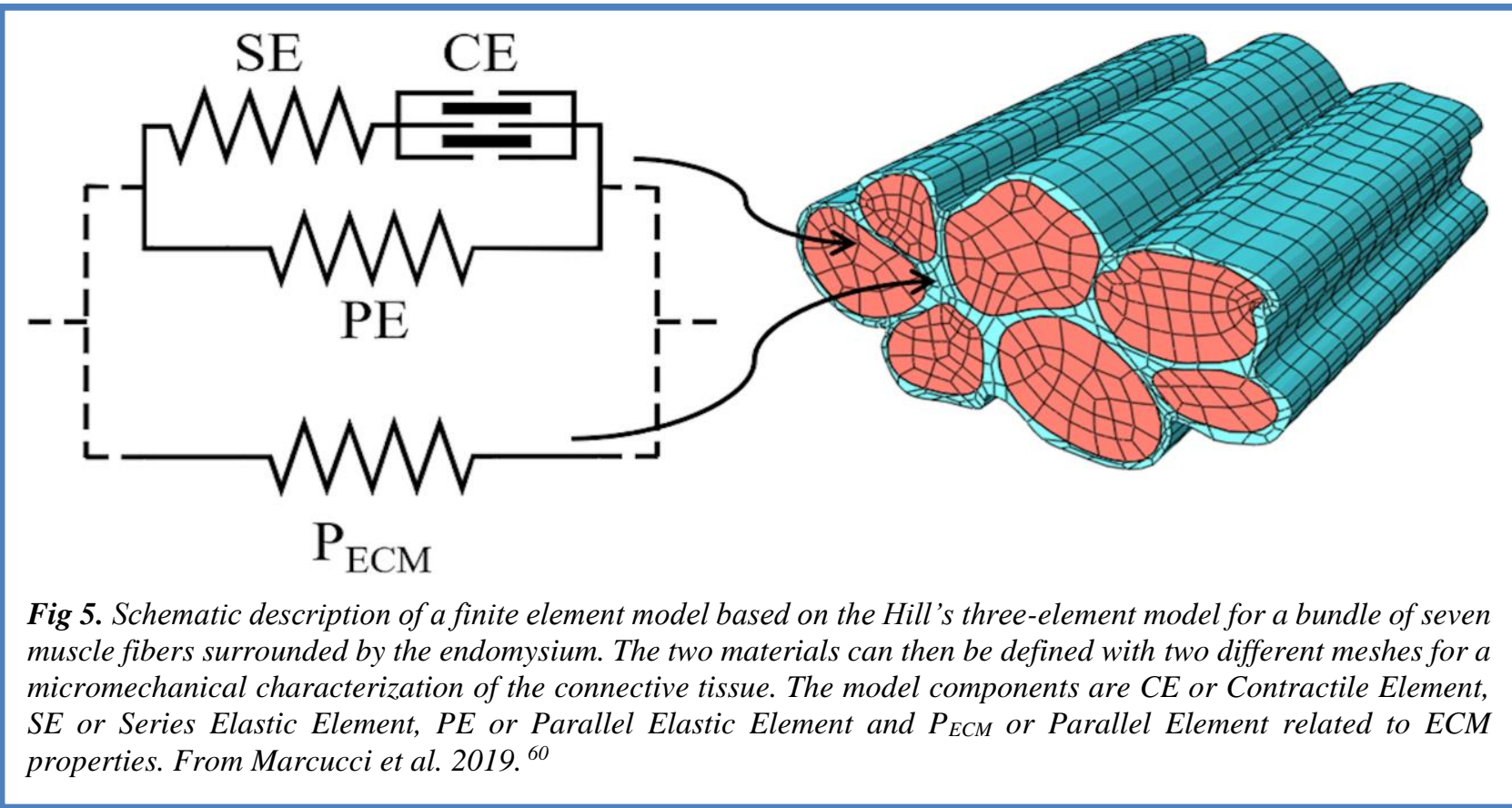

\section{Multiscale modelling of muscle structure}

The assessment of the relative influence of all the agerelated modifications reported above, on the limitation of joint mobility, is a complex problem. In this task, the multiscale modelling of the muscle structure could be an extremely helpful tool. Here we shortly report some of the results obtained in this growing field and our view of its future development. For a quantitative estimation of the tension distribution, a three-dimensional macroscopic model of muscle, which includes fibers and connective tissue, is required. The main active and passive properties in the single fibers are sufficiently well reproduced by the Hill's three-elements model. $^{75}$ Leaving apart more mechanistic models based on the Huxley 1957 original framework,${ }^{76}$ needed to explain new experimental data at the fiber level (see for instance our previous works). ${ }^{77-79}$ Hill's model, which contains a passive elastic element to represents the along-fiber passive component due to intra-sarcomeric proteins, has dominated the field for several decades and is still today a widely used tool to model muscle macroscopically. The Hill's model is very flexible, and several implementations have been made to match more detailed behaviors. ${ }^{80}$ However, it is not based on the microstructure of the muscle. Beside fibers, muscle can be considered as a fiber-reinforced composite material, ${ }^{81}$ and the simplest way to bridge the single fiber model up to the macroscopic, ${ }^{82,83}$ or lower scales ${ }^{84-86}$ is imposing some anisotropy, such as of transversally isotropic hyper elastic materials. However, as noted in Blemker et $\mathrm{al}^{87}$, this approach makes virtually impossible to define a strain energy able to explicitly describe the physical properties of the intramuscular connective tissue. Since a model is reliable as long as its parameters definition is reliable, to analyze the effect of material modification with aging, more detailed microstructural models are required.

We, and others, share the view that not only a detailed description of the fiber component (see Marcucci et al. ${ }^{88}$, but also a precise description of the connective tissue, with an explicitly different material characterization and mesh in the finite element model, ${ }^{60}$ is required to numerically quantify the aging effects on the muscle stiffness (Figure 5). Several models with different degrees of complexity have been proposed in the last two decades. A single fiber surrounded by connective tissue in a simple 2D model to approach the issue of the lateral force transmission mechanism has been presented by Zhang and Gao. ${ }^{89}$ Bleiler et al. ${ }^{90}$ has proposed a microstructurally based bundle-level model where the helical arrangement of the collagen reinforcement is taken into consideration to estimate the effects of the agerelated modifications in the connective tissue. Recently, in Teklemariam et al. an analysis of the effect of different spatial redistribution patterns of activated fibers in young and old subjects has been made, ${ }^{91}$ where fibers and endomysium are modeled with different meshes and with two different hyper elastic constitutive models, showing that the different pattern distribution of force may affect the injury process in aged muscle activation. Separated meshes for the fibers and connective tissue have been used also in Sharafi and Blemker, ${ }^{92}$ showing that higher fiber volume fraction (i.e. smaller relative amount of the connective tissue) transmits higher amounts of force transversely. Preservation in eccentric force in aged muscle, as described in previous sections, has been addressed in the model proposed by Zhang et al. ${ }^{93}$, where a cellular-scale honeycomb-like microstructural muscle model is proposed. Here, differences in age are related to experimental data on rat and mice. Muscles with the 
higher passive stiffness, observed in old mice, generate smaller force in concentric contraction, but an enhanced force in eccentric contraction. Similarly, higher amount of connective tissue in old subjects generates a higher tension in eccentric contraction, but only at high stretches. Beside the characterization of endomysium, similar works are present for epimysium such as in Gao et al. $^{94}$ These kinds of studies, and their future implementations to a macroscopic model, are crucial for our understanding of the problem addressed in this review.

\section{Conclusions}

In conclusion, the available evidences point to an increase of muscle stiffness with aging. The muscletendon complex becomes more rigid and, since tendon stiffness is reduced in elderly, muscle stiffness is expected to increase. Such increase is likely due to change in ECM and connective tissue, although some data on single fibers also suggest a contribution due to increased stiffness of single fibers. The change of muscle stiffness has been considered beneficial as it might contribute to the preservation of eccentric force which has been reported in elderly people. However, the change in muscle stiffness can have a significant impact on the reduction of joint motility. This implies not only reduced range of motion but also the need of greater force to produce the same change of joint angle and this can be a problem in view of the concomitant loss of muscle force of elderly people. In this regard the control of muscle stiffness can be an important target for rehabilitation and physiotherapy, although available evidence suggest that the real impact of stretch training or physiotherapeutic interventions is limited. ${ }^{95}$

\section{List of acronyms}

AGE -.advanced glycation end product

ECM - extracellular matrix

GAG -.glycosaminoglycan

LJM - limitation of joint mobility

\section{Authors contributions}

CR and LM designed the study, analyzed the literature, wrote and approved the paper.

\section{Acknowledgments}

\section{Funding}

LM work is supported by the University of Padova under the MSCA SoE @UniPD programme (Acronym of the project: "Heart Fi-Re", funding: Euros 150.000).

\section{Conflict of Interest}

The authors declare no competing interests.

\section{Ethical Publication Statement}

We confirm that we have read the Journal's position on issues involved in ethical publication and affirm that this report is consistent with those guidelines.

\section{Corresponding Author}

Lorenzo Marcucci, Department of Biomedical Sciences, Padova University, Via Marzolo 3, 35131, Padova, Italy Phone +390498275313 - fax +390498275604.

ORCID iD: 0000-0002-9542-4417

E-mail: lorenzo.marcucci@unipd.it

E-mail of co-author

Carlo Reggiani: carlo.reggiani@unipd.it

ORCID iD: 0000-0001-8080-361X

\section{References}

1 James B, Parker AW. Active and passive mobility of lower limb joints in elderly men and women. Am J Phys Med Rehabil 1989;68:162-7. Doi 10. 1097/00002060-198908000-00002.

2 Roach KE, Miles TP. Normal hip and knee active range of motion: the relationship to age. Phys Ther 1991;71:656-65. Doi 10.1093/ptj/71.9.656.

3 Vandervoort AA, Chesworth BM, Cunningham DA, et al. Age and sex effects on mobility of the human ankle. J Gerontol 1992;47:M17-21. Doi 10. 1093/geronj/47.1.m17.

4 Grimston SK, Nigg BM, Hanley DA, et al. Differences in Ankle Joint Complex Range of Motion as a Function of Age. Foot Ankle 1993;14:215-22. Doi 10.1177/107110079301400 407.

5 Walker JM, Sue D, Miles-Elkousy N, et al. Active mobility of the extremities in older subjects. Phys Ther 1984;64:919-23. Doi 10.1093/ptj/64.6.919.

6 Barnes CJ, Van Steyn SJ, Fischer RA. The effects of age, sex, and shoulder dominance on range of motion of the shoulder. J Shoulder Elbow Surg 2001;10:242-6. Doi 10.1067/mse.2001.115270.

7 Pan F, Arshad R, Zander T, et al. The effect of age and sex on the cervical range of motion. A systematic review and meta-analysis. J Biomech 2018;75:13-27. Doi 10.1016/j.jbiomech.2018.04. 047.

8 Abate M, Carlo LD, Romualdo SD, et al. Postural adjustment in experimental leg length difference evaluated by means of thermal infrared imaging. Physiol Meas 2010;31:35-43. Doi 10.1088/09673334/31/1/003.

9 Wilke J, Macchi V, De Caro R, Stecco C. Fascia thickness, aging and flexibility: is there an association? J Anat. 2019;234:43-9. Doi 10.1111 /joa.12902.

10 Reeves ND, Narici MV, Maganaris CN. Myotendinous plasticity to ageing and resistance exercise in humans. Exp Physiol 2006;91:483-98. Doi 10.1113/expphysiol.2005.032896.

11 Mitchell WK, Williams J, Atherton P, et al. Sarcopenia, Dynapenia, and the Impact of Advancing Age on Human Skeletal Muscle Size and Strength; a Quantitative Review. Front Physiol 2012;3. Doi 10.3389/fphys.2012.00260. 


\section{Age-related increase of resting muscle stiffness}

Eur J Transl Myol 30 (2): 223-233, 2020

12 Clark DJ, Fielding RA. Neuromuscular contributions to age-related weakness. J Gerontol A Biol Sci Med Sci 2012;67:41-7. Doi 10.1093/ gerona/glr041.

13 Ralphs JR, Benjamin M. The joint capsule: structure, composition, ageing and disease. J Anat 1994;184 ( Pt 3):503-9.

14 Tsujii A, Nakamura N, Horibe S. Age-related changes in the knee meniscus. The Knee 2017;24:1262-70. Doi 10.1016/j.knee.2017.08. 001.

15 Kanazawa K, Hagiwara Y, Sekiguchi T, et al. Correlations Between Range of Motion and Elasticity of the Coracohumeral Ligament Evaluated With Shear-Wave Elastography. J Sport Rehabil 2020:1-7. Doi 10.1123/jsr.2019-0279.

16 Yabe Y, Hagiwara Y, Suda H, et al. Joint immobilization induced hypoxic and inflammatory conditions in rat knee joints. Connect Tissue Res 2013;54:210-7. Doi 10.3109/03008207.2013. 786056.

17 Hagiwara Y, Kanazawa K, Ando A, et al. Blood flow changes of the anterior humeral circumflex artery decrease with the scapula in internal rotation. Knee Surg Sports Traumatol Arthrosc 2015;23:1467-72. Doi 10.1007/s00167-013-28232.

18 Toh WS, Brittberg M, Farr J, et al. Cellular senescence in aging and osteoarthritis. Acta Orthop 2016;87:6-14. Doi 10.1080/17453674.2016.123 5087.

19 Loeser RF. Aging and Osteoarthritis: The Role of Chondrocyte Senescence and Aging Changes in the Cartilage Matrix. Osteoarthritis Cartilage 2009;17:971-9. Doi: 10.1016/j.joca.2009.03.002.

20 Maganaris CN, Narici MV, Reeves ND. In vivo human tendon mechanical properties: effect of resistance training in old age. J Musculoskelet Neuronal Interact 2004;4:204-8.

21 Onambele GL, Narici MV, Maganaris CN. Calf muscle-tendon properties and postural balance in old age. J Appl Physiol (1985) 2006;100:2048-56. Doi 10.1152/japplphysiol.01442.2005.

22 Alnaqeeb MA, Al Zaid NS, Goldspink G. Connective tissue changes and physical properties of developing and ageing skeletal muscle. J Anat 1984;139( Pt 4):677-89.

23 Wood LK, Kayupov E, Gumucio JP, et al. Intrinsic stiffness of extracellular matrix increases with age in skeletal muscles of mice. J Appl Physiol (1985) 2014;117:363-9. Doi 10.1152/japplphysiol.00256. 2014.

24 Gosselin LE, Adams C, Cotter TA, et al. Effect of exercise training on passive stiffness in locomotor skeletal muscle: role of extracellular matrix. J Appl Physiol (1985) 1998;85:1011-6. Doi 10.1152/jappl. 1998 .85.3.1011.
25 Brown M, Fisher JS, Salsich G. Stiffness and muscle function with age and reduced muscle use. J Orthop Res Off Publ Orthop Res Soc 1999;17:40914. Doi 10.1002/jor.1100170317.

26 Blanpied P, Smidt GL. The difference in stiffness of the active plantarflexors between young and elderly human females. J Gerontol 1993;48:M58-63. Doi 10.1093/geronj/48.2.m58.

27 Palmer TB, Thompson BJ. Influence of age on passive stiffness and size, quality, and strength characteristics. Muscle Nerve 2017;55:305-15. Doi 10.1002/mus.25231.

28 Ochala J, Lambertz D, Pousson M, et al. Changes in mechanical properties of human plantar flexor muscles in ageing. Exp Gerontol 2004;39:349-58. Doi 10.1016/j.exger.2003.11.004.

29 Valour D, Pousson M. Compliance changes of the series elastic component of elbow flexor muscles with age in humans. Pflugers Arch 2003;445:7217. Doi 10.1007/s00424-002-0871-4.

30 Sobolewski EJ, Ryan ED, Thompson BJ, et al. The influence of age on the viscoelastic stretch response. J Strength Cond Res 2014;28:1106-12. Doi 10.1519/JSC.0000000000000326.

31 Alfuraih AM, Tan AL, O'Connor P, et al. The effect of ageing on shear wave elastography muscle stiffness in adults. Aging Clin Exp Res 2019; 31:1755-63. Doi 10.1007/s40520-019-01139-0.

32 Vandervoort AA, Kramer JF, Wharram ER. Eccentric knee strength of elderly females. J Gerontol 1990;45:B125-128. Doi 10.1093/geronj/ 45.4.b125.

33 Hortobágyi T, Zheng D, Weidner M, et al. The influence of aging on muscle strength and muscle fiber characteristics with special reference to eccentric strength. J Gerontol A Biol Sci Med Sci 1995;50:B399-406. Doi 10.1093/gerona/50a.6.b 399.

34 Roig M, Macintyre DL, Eng JJ, et al. Preservation of eccentric strength in older adults: Evidence, mechanisms and implications for training and rehabilitation. Exp Gerontol 2010;45:400-9. Doi 10.1016/j.exger.2010.03.008.

35 Macaluso A, Nimmo MA, Foster JE, et al. Contractile muscle volume and agonist-antagonist coactivation account for differences in torque between young and older women. Muscle Nerve 2002;25:858-63. Doi 10.1002/mus.10113.

36 Hortobágyi T, Devita P. Mechanisms responsible for the age-associated increase in coactivation of antagonist muscles. Exerc Sport Sci Rev 2006;34:29-35. Doi 10.1097/00003677-200601000 -00007 .

37 Lindstedt SL, LaStayo PC, Reich TE. When active muscles lengthen: properties and consequences of eccentric contractions. News Physiol Sci 2001;16:256-61. Doi 10.1152/physiologyonline. 2001.16.6.256. 
38 Ochala J, Dorer DJ, Frontera WR, et al. Single skeletal muscle fiber behavior after a quick stretch in young and older men: a possible explanation of the relative preservation of eccentric force in old age. Pflugers Arch 2006;452:464-70. Doi 10.1007/ s00424-006-0065-6.

39 Ochala J, Frontera WR, Dorer DJ, et al. Single skeletal muscle fiber elastic and contractile characteristics in young and older men. J Gerontol A Biol Sci Med Sci 2007;62:375-81. Doi 10.1093/ gerona/62.4.375.

40 LaStayo PC, Woolf JM, Lewek MD, et al. Eccentric muscle contractions: their contribution to injury, prevention, rehabilitation, and sport. J Orthop Sports Phys Ther 2003;33:557-71. Doi 10.2519/ jospt.2003.33.10.557.

41 Hortobágyi T, DeVita P. Favorable neuromuscular and cardiovascular responses to 7 days of exercise with an eccentric overload in elderly women. J Gerontol A Biol Sci Med Sci 2000;55:B401-10. Doi 10.1093/gerona/55.8.b401.

42 Trappe S, Gallagher P, Harber M, et al. Single Muscle Fibre Contractile Properties in Young and Old Men and Women. J Physiol 2003;552:47-58. Doi 10.1113/jphysiol.2003.044966.

43 D'Antona G, Pellegrino MA, Adami R, et al. The effect of ageing and immobilization on structure and function of human skeletal muscle fibres. J Physiol 2003;552:499-511. Doi 10.1113/jphysiol. 2003.046276.

44 Lim JY, Choi SJ, Widrick JJ, et al. Passive force and viscoelastic properties of single fibers in human aging muscles. Eur J Appl Physiol 2019;119:233948. Doi 10.1007/s00421-019-04221-7.

45 Fridén J, Lieber RL. Spastic muscle cells are shorter and stiffer than normal cells. Muscle Nerve 2003. Doi 10.1002/mus.10247.

46 Olsson MC, Krüger M, Meyer L-H, et al. Fibre type-specific increase in passive muscle tension in spinal cord-injured subjects with spasticity. J Physiol 2006;577:339-52. Doi 10.1113/jphysiol. 2006.116749.

47 Prado LG, Makarenko I, Andresen C, et al. Isoform diversity of giant proteins in relation to passive and active contractile properties of rabbit skeletal muscles. J Gen Physiol 2005;126:461-80. Doi 10.1085/jgp.200509364.

48 Schiaffino S, Reggiani C. Fiber types in mammalian skeletal muscles. Physiol Rev 2011;91:1447-531. Doi 10.1152/physrev.00031.2010.

49 Wang K, McCarter R, Wright J, et al. Regulation of skeletal muscle stiffness and elasticity by titin isoforms: a test of the segmental extension model of resting tension. Proc Natl Acad Sci U S A 1991;88:7101-5.

50 Horowits R. Passive force generation and titin isoforms in mammalian skeletal muscle. Biophys $\mathbf{J}$ 1992;61:392-8.
51 Mutungi G, Trinick J, Ranatunga KW. Resting tension characteristics in differentiating intact rat fast- and slow-twitch muscle fibers. J Appl Physiol (1985) 2003;95:2241-7. Doi 10.1152/ japplphysiol. 00990.2002.

52 Freundt JK, Linke WA. Titin as a force-generating muscle protein under regulatory control. J Appl Physiol (1985) 2019;126:1474-82. Doi 10.1152/ japplphysiol.00865.2018.

53 Labeit D, Watanabe K, Witt C, et al. Calciumdependent molecular spring elements in the giant protein titin. Proc Natl Acad Sci U S A 2003; 100:13716-21. Doi 10.1073/pnas.2235652100.

54 Kjaer M. Role of extracellular matrix in adaptation of tendon and skeletal muscle to mechanical loading. Physiol Rev 2004;84:649-98. Doi 10.1152 /physrev.00031.2003.

55 Borg TK, Caulfield JB. Morphology of connective tissue in skeletal muscle. Tissue Cell 1980;12:197207. Doi 10.1016/0040-8166(80)90061-0.

56 Street SF. Lateral transmission of tension in frog myofibers: a myofibrillar network and transverse cytoskeletal connections are possible transmitters. J Cell Physiol 1983;114:346-64. Doi 10.1002/jcp. 1041140314.

57 Huijing PA, Baan GC, Rebel GT. Nonmyotendinous force transmission in rat extensor digitorum longus muscle. J Exp Biol 1998;201:683-91.

58 Meyer GA, Lieber RL. Elucidation of extracellular matrix mechanics from muscle fibers and fiber bundles. J Biomech 2011;44:771-3. Doi 10.1016 /j.jbiomech.2010.10.044.

59 Meyer G, Lieber RL. Muscle fibers bear a larger fraction of passive muscle tension in frogs compared with mice. J Exp Biol 2018;221: jeb182089. Doi 10.1242/jeb.182089.

60 Marcucci L, Bondì M, Randazzo G, et al. Fibre and extracellular matrix contributions to passive forces in human skeletal muscles: An experimental based constitutive law for numerical modelling of the passive element in the classical Hill-type three element model. PLOS ONE 2019;14:e0224232. Doi 10.1371/journal.pone.0224232.

61 Kragstrup TW, Kjaer M, Mackey AL. Structural, biochemical, cellular, and functional changes in skeletal muscle extracellular matrix with aging. Scand J Med Sci Sports 2011;21:749-57. doi 10.1111/j.1600-0838.2011.01377.x.

62 Gao Y, Kostrominova TY, Faulkner JA, et al. Agerelated changes in the mechanical properties of the epimysium in skeletal muscles of rats. $\mathrm{J}$ Biomech 2008;41:465-9. Doi 10.1016/j.jbiomech.2007.09. 021.

63 Delabastita T, Bogaerts S, Vanwanseele B. AgeRelated Changes in Achilles Tendon Stiffness and Impact on Functional Activities: A Systematic 
Review and Meta-Analysis. J Aging Phys Act 2018:1-12. Doi 10.1123/japa.2017-0359.

64 Eriksen CS, Henkel C, Svensson RB, et al. Lower tendon stiffness in very old compared with old individuals is unaffected by short-term resistance training of skeletal muscle. J Appl Physiol (1985) 2018;125:205-14. Doi 10.1152/ japplphysiol. 00028.2018.

65 Hsiao M-Y, Chen Y-C, Lin C-Y, et al. Reduced Patellar Tendon Elasticity with Aging: In Vivo Assessment by Shear Wave Elastography. Ultrasound Med Biol 2015;41:2899-905. Doi 10.1016/j.ultrasmedbio.2015.07.008.

66 Riley GP, Harrall RL, Constant CR, et al. Glycosaminoglycans of human rotator cuff tendons: changes with age and in chronic rotator cuff tendinitis. Ann Rheum Dis 1994;53:367-76. Doi 10.1136/ard.53.6.367.

67 Vogel HG. Age dependence of mechanical properties of rat tail tendons (hysteresis experiments). Aktuelle Gerontol 1983;13:22-7.

68 Nakagawa Y, Hayashi K, Yamamoto N, et al. Agerelated changes in biomechanical properties of the Achilles tendon in rabbits. Eur J Appl Physiol 1996;73:7-10. Doi 10.1007/bf00262803.

69 Dressler MR, Butler DL, Wenstrup R, et al. A potential mechanism for age-related declines in patellar tendon biomechanics. J Orthop Res 2002;20:1315-22. Doi 10.1016/S0736-0266(02) 00052-9.

70 Slane LC, Martin J, DeWall R, et al. Quantitative ultrasound mapping of regional variations in shear wave speeds of the aging Achilles tendon. Eur Radiol 2017;27:474-82. Doi 10.1007/s00330-0164409-0.

71 Svensson RB, Heinemeier KM, Couppé C, et al. Effect of aging and exercise on the tendon. J Appl Physiol (1985) 2016;121:1237-46. Doi 10.1152/ japplphysiol.00328.2016.

72 Stecco C. Functional Atlas of the Human Fascial System E-Book. Elsevier Health Sciences; 2014.

73 Stecco C, Tiengo C, Stecco A, et al. Fascia redefined: anatomical features and technical relevance in fascial flap surgery. Surg Radiol Anat 2013;35:369-76. Doi 10.1007/s00276-012-1058-0.

74 Trindade VLA, Martins PA, Santos S, et al. Experimental study of the influence of senescence in the biomechanical properties of the temporal tendon and deep temporal fascia based on uniaxial tension tests. J Biomech 2012;45:199-201. Doi 10. 1016/j.jbiomech.2011.09.018.

75 Kojic M, Mijailovic S, Zdravkovic N. Modelling of muscle behaviour by the finite element method using Hill's three-element model. Int $\mathrm{J}$ Numer Methods Eng 1998;43:941-53. Doi 10.1002/ (SICI)1097-0207 (19981115)43:5<941:: AIDNME435>3.0.CO;2-3.
76 Huxley AF. Muscle Structure and Theories of Contraction. Prog Biophys Biophys Chem 1957;7:255-318.

77 Marcucci L, Reggiani C. Mechanosensing in Myosin Filament Solves a 60 Years Old Conflict in Skeletal Muscle Modeling between High Power Output and Slow Rise in Tension. Front Physiol 2016;7:427. Doi 10.3389/fphys.2016.00427.

78 Marcucci L, Washio T, Yanagida T. Titin-mediated thick filament activation, through a mechanosensing mechanism, introduces sarcomere-length dependencies in mathematical models of rat trabecula and whole ventricle. Sci Rep 2017;7:5546. Doi 10.1038/s41598-017-05999-2.

79 Marcucci L, Washio T, Yanagida T. Proposed mechanism for the length dependence of the force developed in maximally activated muscles. Sci Rep 2019;9:1317. Doi 10.1038/s41598-018-36706-4.

80 Epstein M (Marcelo). Theoretical models of skeletal muscle: biological and mathematical considerations. Chichester New York: Wiley; 1998.

81 Huijing PA. Muscle as a collagen fiber reinforced composite: a review of force transmission in muscle and whole limb. J Biomech 1999;32:329-45. Doi 10.1016/s0021-9290(98)00186-9.

82 Pato MPM, Santos NJG, Areias P, et al. Finite element studies of the mechanical behaviour of the diaphragm in normal and pathological cases. Comput Methods Biomech Biomed Engin 2011;14:505-13. Doi 10.1080/10255842.2010.48 3683.

83 Zhang G, Chen X, Ohgi J, et al. Biomechanical simulation of thorax deformation using finite element approach. Biomed Eng OnLine 2016;15:118. Doi 10.1186/s12938-016-0132-y.

84 Johansson T, Meier P, Blickhan R. A FiniteElement Model for the Mechanical Analysis of Skeletal Muscles. J Theor Biol 2000;206:131-49. Doi 10.1006/jtbi.2000.2109.

85 Martins JAC, Pires EB, Salvado R, et al. A numerical model of passive and active behavior of skeletal muscles. Comput Methods Appl Mech Eng 1998;151:419-33. Doi 10.1016/S0045-7825(97) 00162-X.

86 Oomens CWJ, Maenhout M, van Oijen $\mathrm{CH}$, et al. Finite element modelling of contracting skeletal muscle. Philos Trans R Soc Lond B Biol Sci 2003;358:1453-60. Doi 10.1098/rstb.2003.1345.

87 Blemker SS, Pinsky PM, Delp SL. A 3D model of muscle reveals the causes of nonuniform strains in the biceps brachii. J Biomech 2005;38:657-65. Doi 10.1016/j.jbiomech.2004.04.009.

88 Marcucci L, Reggiani C, Natali AN, et al. From single muscle fiber to whole muscle mechanics: a finite element model of a muscle bundle with fast and slow fibers. Biomech Model Mechanobiol 2017;16:1833-43. Doi 10.1007/s10237-017-0922-6. 


\section{Age-related increase of resting muscle stiffness}

Eur J Transl Myol 30 (2): 223-233, 2020

Zhang C, Gao Y. Finite element analysis of mechanics of lateral transmission of force in single muscle fiber. J Biomech 2012;45:2001-6. Doi 10.1016/j.jbiomech.2012.04.026.

90 Bleiler C, Ponte Castañeda P, Röhrle O. A microstructurally-based, multi-scale, continuummechanical model for the passive behaviour of skeletal muscle tissue. J Mech Behav Biomed Mater 2019;97:171-86. Doi 10.1016/j.jmbbm.2019.05.012.

91 Teklemariam A, Hodson-Tole E, Reeves ND, et al. A micromechanical muscle model for determining the impact of motor unit fiber clustering on force transmission in aging skeletal muscle. Biomech Model Mechanobiol 2019;18:1401-13. Doi 10.100 7/s10237-019-01152-2.

92 Sharafi B, Blemker SS. A mathematical model of force transmission from intrafascicularly terminating muscle fibers. J Biomech 2011; 44:2031-9. Doi 10.1016/j.jbiomech.2011.04. 038.
93 Zhang Y, Chen J-S, He Q, et al. Microstructural analysis of skeletal muscle force generation during aging. Int $\mathbf{J}$ Numer Methods Biomed Eng 2020;36:e3295. Doi 10.1002/cnm.3295.

94 Gao Y, Wineman AS, Waas AM. Mechanics of muscle injury induced by lengthening contraction. Ann Biomed Eng 2008;36:1615-23. doi 10.100 7/s10439-008-9547-3.

95 Blazevich AJ. Adaptations in the passive mechanical properties of skeletal muscle to altered patterns of use. J Appl Physiol (1985) 2019;126:1483-91. Doi 10.1152/japplphysiol.0070 0.2018 .

Submitted: March 25, 2020

Revision received: April 6, 2020 Accepted for publication: April 6, 2020 